\title{
PROPOSAL FOR A TIME-DEPENDENT DYNAMIC IDENTIFICATION ALGORITHM FOR STRUCTURAL HEALTH MONITORING
}

\author{
MANUEL F. HORMAZÁBAL ${ }^{1 *}$, MARIA GIOVANNA MASCIOTTA ${ }^{2}$, \\ DANIEL V. OLIVEIRA ${ }^{1}$ \\ ${ }^{1}$ Institute for Sustainability and Innovation in Structural Engineering (ISISE) \\ Institute of Science and Innovation for Bio-Sustainability (IB-S) \\ Department of Civil Engineering \\ University of Minho \\ Campus de Azurém, 4800-058 Guimarães, Portugal

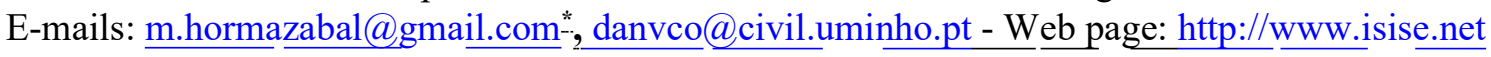 \\ ${ }^{2}$ Department of Engineering and Geology (InGeo) \\ G. d'Annunzio University of Chieti-Pescara \\ Viale Pindaro 42, 65127, Pescara, Italy \\ E-mail: g.masciotta@unich.it - Web page: http://www.ingeo.unich.it
}

Keywords: Structural Health Monitoring, Non-Destructive Inspection, Operational Modal Analysis, Time-dependent, Structural Dynamic Identification.

\begin{abstract}
This paper describes the design, test and validation processes of a dynamic identification algorithm aimed at the time-dependent assessment of modern structures and heritage buildings for civil and seismic engineering purposes. Full validation of the algorithm is performed through analysis of numerically simulated data from an idealized masonry tower. Making use of output-only vibration measurements, the non-parametric algorithm can generate dynamic features results as time-dependent functions for the complete observation period. The algorithm can work in the presence of different dynamic loads and non-linear structural behaviours, close spectral frequency components and noisecontaminated data. Time-dependent structural dynamic parameters that can be computed are modal frequencies, modal displacements, modal curvatures, and higher derivatives of mode shapes. The proposed algorithm aims to be used as the core estimator of timedependent identification methods devoted to the health monitoring of structures and infrastructures, being suitable for a multitude of tasks ranging from the simple operational modal analysis (in pre and post-event condition) to the complex online assessment of the structural response during seismic events for rapid damage identification.
\end{abstract}

\section{INTRODUCTION}

In order to appropriately plan maintenance and conservation actions in existing structures and heritage buildings, the execution of field dynamic testing under operational conditions supported by periodic Structural Health Monitoring (SHM) campaigns has become imperative 
$[1,2,3]$. Traditional dynamic identification methods used in Operational Modal Analysis (OMA) can accurately estimate parameters like modal frequencies, damping ratios and mode shapes $[4,5,6]$, but some methods present problems related to the difficulties in identifying close-spaced modes or uncertainties when working with noise-contaminated measurements $[7,8,9]$. On the other hand, most methods based on output-only data work with parametric eigenvalue decompositions of a weighted data matrix, like the Singular Value Decomposition (SVD) of the Power Spectral Density matrix (PSD) - as far as the Frequency Domain Decomposition (FDD) methods are concerned [10,11,12] - or the SVD of Hankel matrixes in case of Stochastic System Identification methods (SSI) $[13,14,15]$. Thus, they are restricted to the linear-elastic range (no-damage, no-yielding) and are not suitable to identify dynamic features in the presence of nonlinearities. Moreover, these methods cannot generate results as time-dependent functions since they are limited to the comparative assessment between different structural conditions (usually before and after a particular event), thereby being unable to provide any information about the actual temporal evolution of dynamic parameters like natural frequencies and mode shapes during the damage progress. To effectively upgrade stateof-the-art dynamic identification techniques for SHM intents, performing dynamic identification in the presence of nonlinearities and tracking relevant time-dependent modal parameters are essential tasks to accomplish $[16,17,18,19]$. The development, testing and preliminary validation of a non-parametric algorithm suitable for this purpose are hereby presented. The proposed algorithm is capable of processing linear (no damage) and nonlinear (with damage) data and can properly conduct dynamic identification either with forced accelerations measured during scismic events or with random data coming from ambient vibration tests. Results are generated as time-dependent functions, allowing evolution of dynamic parameters before, during and after the occurrence of
algorithm aims to be used as the core estimator of vibration-based structural heat tools in tasks ranging from the simple OMA for pre- and post-event analysis online assessment of the structural response during seismic events for rapid damage buildings,

\section{BRIEF THEORETICAL FRAMEWORK}

\subsection{Wavelet Transform}

Mathematically, the Wavelet Transform (WT) or wavelet Time-Frequency Analysis (TFA) is an integral transform that represents a signal $x(t)$ in terms of a series of coefficients related to convolutions of the signal data with dilated and translated versions of a compactly supported wavelet function $\psi(\cdot)[20]$. The Continuous Wavelet Transform (CWT) is one of the most widely high-resolution TFA, and it has been extensively exploited for a variety of purposes [21]-[24]. The CWT of a signal $x(t)$ is mathematically defined as:

$$
\operatorname{CWT}\{x(t)\}=|a|^{-1 / 2} \int_{-\infty}^{\infty} x(t) \psi\left(\frac{t-b}{a}\right) d t
$$

where a indicates the dilatation factor, $\mathrm{b}$ the translation factor, and $\psi(\cdot)$ is the wavelet function. 
In the discrete version of the WT, namely the Discrete Wavelet Transform (DWT), the wavelet function and the dilatation and translation factors are discretized in order to compute the wavelet coefficients only on specific time instants and at specific frequency scales. A discrete wavelet $\psi_{n, m}(s)$ is defined as:

$$
\psi_{n, m}(s)=a_{0}{ }^{m / 2} \tilde{\psi}\left(a_{0}{ }^{-m} s-n b_{0}\right)
$$

in which $\left\{s: \psi_{n, m}(s) \neq 0\right\}$ is the set that supports the wavelet function, whereas $a=a_{0}^{m}$ $\left\{\forall a \in \mathbb{R}_{+}: a \neq 0\right\}$ is the discrete dilatation coefficient, and $b=n b_{0} a_{0}^{m}\{\forall b \in \mathbb{R}\}$ is the discrete translation coefficient. If required, the original sequence $x[n]$ can be fully reconstructed through the following equations:

$$
\begin{gathered}
x[n]=\sum_{n, m}\left\langle x, \psi_{n, m}\right\rangle \hat{\psi}_{n, m} \\
\hat{\psi}_{n, m}(s)=a_{0}{ }^{-m / 2} \psi^{*}\left(a_{0}{ }^{-m_{S}}-n\right)
\end{gathered}
$$

\subsection{Discrete Wavelet Transform Decompositions}

Equations (3) and (4) shed light on the idea that DWT represents the discrete signal $x[n]$ through sets of coefficients computed through convolutions of $x[n]$ with a set of properly selected filters. According to the previous, on a discrete wavelet decomposition algorithm a new subsequence of dati filter $g_{m}[n]$ as the scal where $m$ is the number corresponding pair of convolution: the Approximation Coefficients
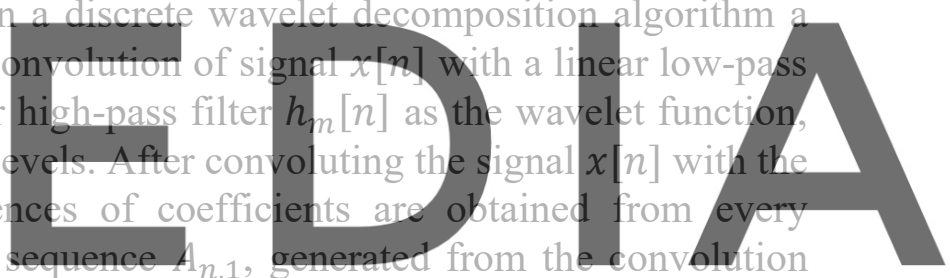

with low-pass filter $g_{n, 1}[n]$, and the Details coefficients sequence $D_{n, 1}$, generated from the

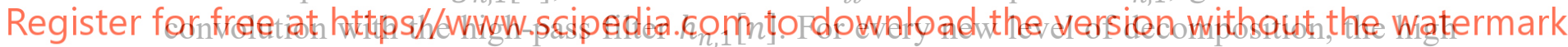

half of the frequency spectrum is discarded, so according to Nyquist-Shannon criteria $[25,26]$ the original number of samples will be redundant. Due to this, a decimation of $A_{n, 1}$ and $D_{n, 1}$ is performed in each level of decomposition, so every subsequence will have half the samples of his immediate superior parent sequence. The convolution of the filters impulse responses with the signal will apply repeatedly until reaching maximum level, decomposing the signal in the form of a cascade serial processing algorithm, where every new $D_{n, m}[n]$ sequence obtained is decomposed into a pair of new $A_{n, m+1}[n]$ and $D_{n, m+1}[n]$ subsequences. If an orthogonal mother wavelet function is selected, all the generated sub-sequences will be also orthogonal, thus, they will keep the energy distribution according to the original data.

\subsection{Maximum Overlap Discrete Wavelet Packet Transform}

In the Maximum Overlap Discrete Wavelet Packet Transform (MODWPT) [27], there is no decimation applied to the original data nor any $W_{n, m}$ subsequence, thus all $W_{n, m}$ sub-sequences will maintain the same number of samples than the original stream of data and there will be no 
loss of resolution on low frequencies. The original spectrum is sliced into $2^{m}$ discrete frequency bands, each one corresponding to a family of packets of mother wavelets and scale functions.

\subsection{Instantaneous frequency through Hilbert transform}

The Hilbert Transform (HT) is defined as the convolution of $x(t)$ with the function $g(t)=$ $1 / \pi t$ and it is essential for constructing analytic signals. The Analytic Signal (AS) is the representation of any signal or data stream as a complex pair where the imaginary part is the Hilbert transform of the real-valued signal $x(t)$. By the modulus operation, the Instantaneous Amplitude is calculated, and by the time derivative of the analytic signal's complex angle, the Instantaneous Frequency is obtained. The Hilbert spectrum is a graphical representation of the instantaneous frequency, the instantaneous amplitude and the time evolution of the signal, usually, in the form of a spectrograph of instantaneous frequency vs time with the instantaneous amplitude in the colour scale. The Hilbert transform of a discrete Gaussian white noise will produce as many different instantaneous frequency values as the number of samples of the signal. Due to the previous, broadband signals are not good candidates for Hilbert spectrum analysis and it is better to decompose or band filter the raw data before any further analysis or process with HT.

\section{METHODS AND MATERIALS}

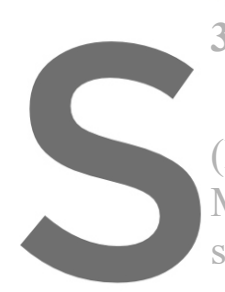

\subsection{Algorithm description}

The Enhanced Moclal Identification for Long-Term (EMILIA) proposed in

MODWPT decompositi

sequences from raw Multi

measurements. MODWPT was selected due to

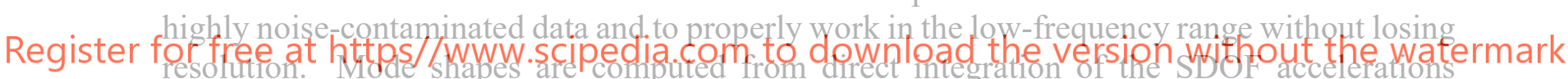

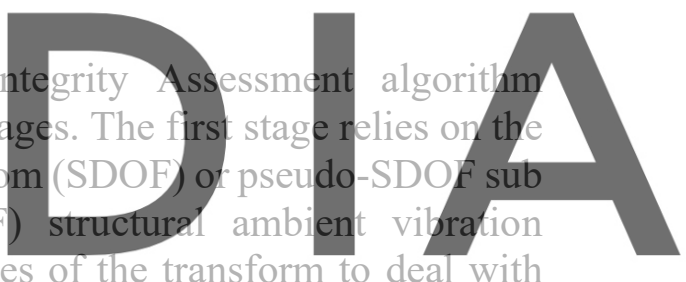

computed by the wavelet decomposition (this step is not necessary if displacement measurements are analysed). In the second stage, the Hilbert Transform is applied to each pseudo-SDOF to compute the analytical signals and subsequently obtain the instantaneous frequency and instantaneous amplitude functions. As the instantaneous frequency is computed through the time derivative of the oscillatory phase, it follows that the instantaneous frequency is a time-dependent parameter. Ambient structural vibration measurements have deterministic data well-hidden between stochastic data. Due to this, the initial Wavelet decomposition is of critical importance to analyse ambient vibrations with Hilbert transform. The capability of this integral transform to compute a frequency value for each sample of the analysed signal is used as an intent to reduce the influence of uncertainties. A deep analysis of these aspects can be found in [28]. Final computations involve statistical analyses. Probability Density Functions (PDFs) with a Kernel distribution are computed for each instantaneous frequency function to identify the values with higher probabilities and their corresponding damping factors. PDF resorts to a supposed probability data distribution to fit the data, where the most commonly 
used is the parametric Gaussian normal distribution. Such a distribution can be found in most measured data with a universe bigger than thirty samples, nevertheless, the previous does not apply to the frequency data distribution of an undamped structural mode featuring a resonant peak that goes over the maximum value given by a normal distribution, thus the frequency peak bandwidth is also narrower. This could lead to overestimations on damping factor values for each modal component. To overcome this issue, Kernel distribution was chosen due to the wellfitting capabilities of assessing data which is highly contaminated with aleatory variables. Further information about Kernel distribution can be found in [29]. The schematics for the complete algorithm are presented in Figure 1.
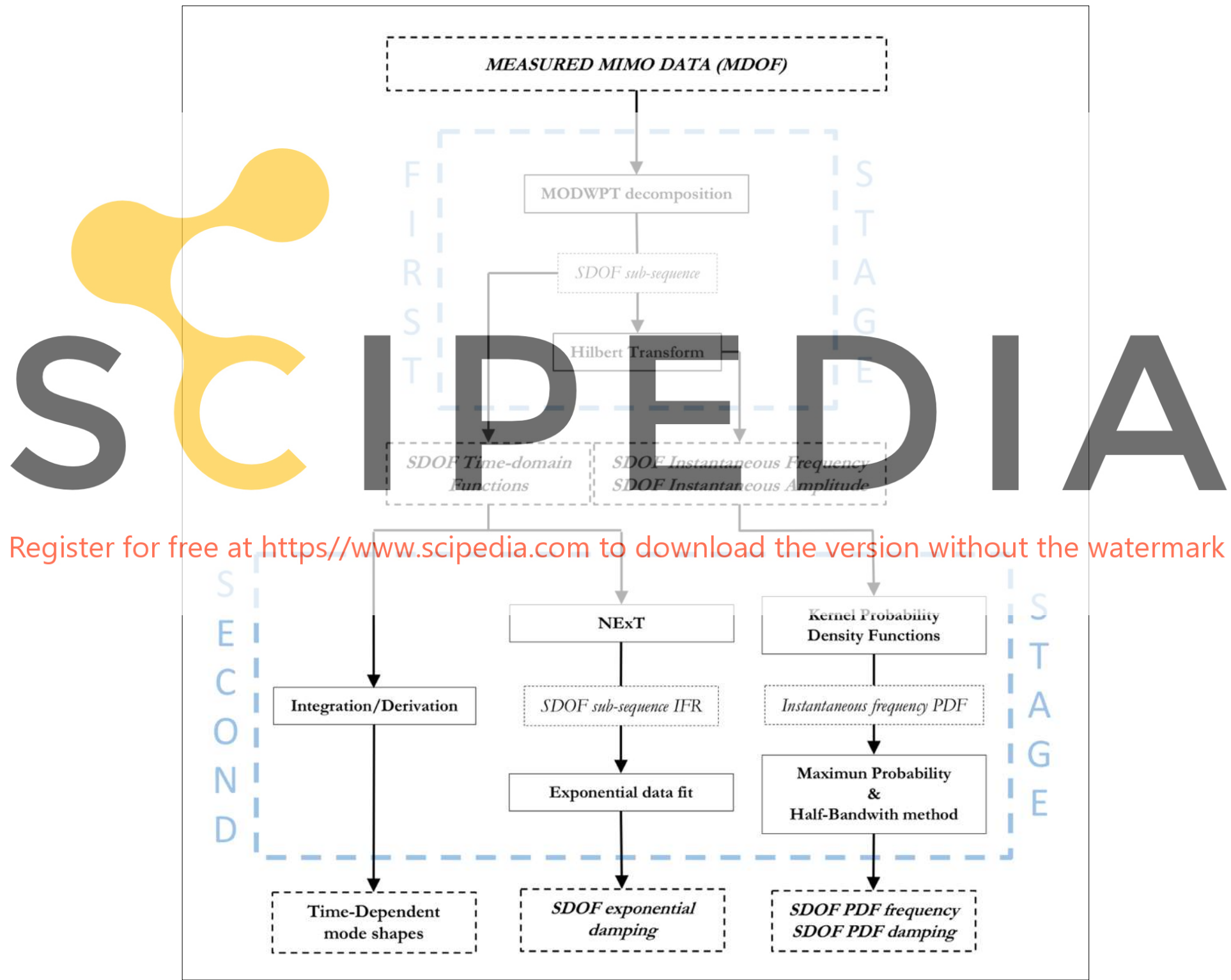

Figure 1: Proposed algorithm schematic. 


\subsection{FEM-based validation}

A Finite Element Model (FEM) of an idealized masonry tower was developed in Diana FEA $^{\circledR}$ software in order to numerically validate the proposed algorithm. The model was built resorting exclusively to volumetric elements. Concerning the morphological aspects, the tower features a square plan of $6 \mathrm{~m}$ side, with double-leaf masonry walls of $0.6 \mathrm{~m}$ thickness, two timber floors $10 \mathrm{~m}$ and $20 \mathrm{~m}$ high, respectively, and a total height of $28 \mathrm{~m}$. Floor slabs are directly connected to the timber beams inserted in the masonry wall thickness. The material properties adopted for the numerical model are reported in . It is noted that a linear behaviour was assumed for all timber elements, whereas nonlinear properties were assigned to the masonry material. Particularly, the latter were set based on values retrieved from the literature $[30]$.

Table 1: Material properties adopted for the numerical model.

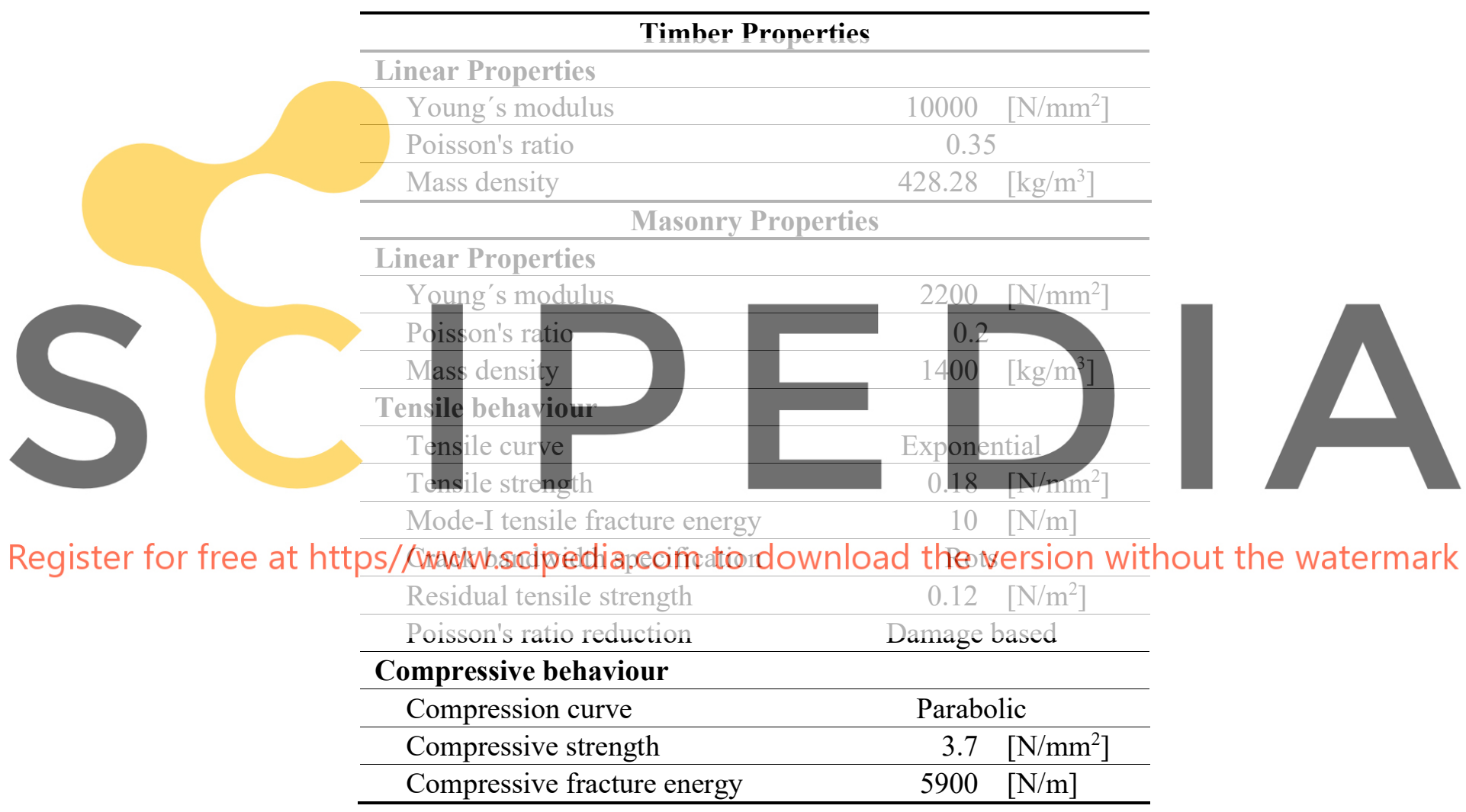

Interaction between tower foundations and soil was modelled as a boundary interface connection with Coulomb friction with cohesion of $100 \mathrm{kN} / \mathrm{m}$, that was used between the model base elements and the ground fixed supports. The FE model counted a total of 8467 quadratic/hexagonal elements with quadratic mesh order and linear mid-side point interpolation. Elements maximum side length was $0.5 \mathrm{~m}$. A Total-Lagrange geometrical formulation and crack-model based on total strain were used. For an initial understanding of the tower's dynamic behaviour, a free vibration eigenvalue analysis was conducted first. This 
allowed to properly define the acquisition parameters necessary for the subsequent TimeHistory Non-Linear analyses (THNL) as well as the number and position of measurement points to guarantee a sufficient spatial density of the system's vibration response under simulated ambient noise and real seismic inputs. THNL analyses were performed using the HilbertHughes-Taylor (HHT) transient time integration with $\alpha=-0,1$ through the Secant iteration method, setting $\Delta t=0.01 \mathrm{~s}$ (see also section 4.1). Requested data outputs were the accelerations in $\mathrm{X}, \mathrm{Y}$ and $\mathrm{Z}$ directions. The first THNL analysis was conducted by randomly applying Gaussian white noise excitations to different points of the structure. The second THNL analysis was performed using the same white noise excitations from first analysis in combination with a real earthquake signal applied as base excitation in all three directions. The ground motion selected for this purpose was the Loma Prieta earthquake of magnitude $\mathrm{M}_{\mathrm{w}}=7.0$ that occurred on October $18^{\text {th }}, 1989$ and was recorded at the CGS-CSMIP Station 47381, located on a sewer farm around $31.1 \mathrm{~km}$ far from the epicentre. Data were sampled at $0.01 \mathrm{~s}$ for a total duration of 39.9 seconds, resulting into 3991 data points. The earthquake featured a PGA equal to $0.5 \mathrm{~g}$ and a maximum displacement of $0.1 \mathrm{~m}$ (Y-axis). From each corner of the tower 15 nodes equally spaced in height each $2 \mathrm{~m}$ were selected along the four edges of the tower to acquire its nodal response in terms of accelerations. All nodal processes collected in X and Y directions were then loaded into the core algorithm coded in MATLAB ${ }^{\circledR}$ as well as into ARTeMIS ${ }^{\circledR}$ software for output-only modal analysis with traditional estimators.
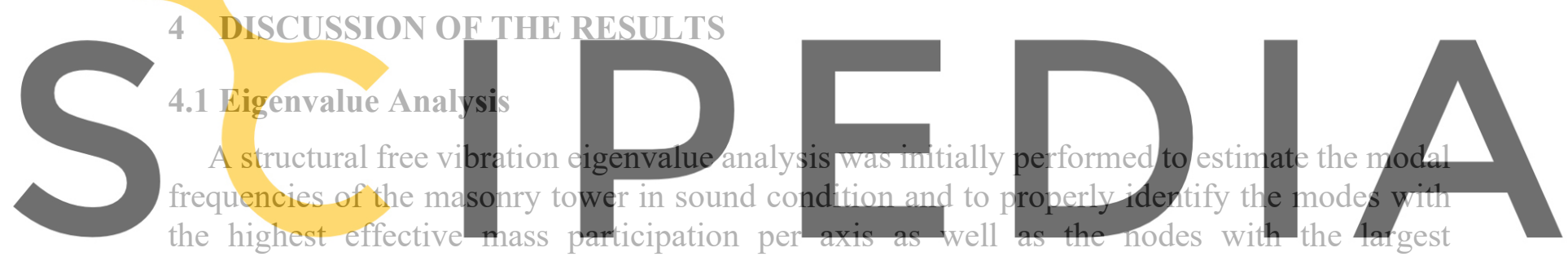

displacement per mode. The modal periods, frequencies and deformed shapes of the first six

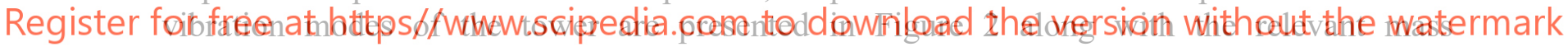
participation. From the results, it was concluded that the dynamic response of the tower could be adequately described by the first six vibration modes since the accumulated participating mass in the frequency range $1-12 \mathrm{~Hz}$ was about $85 \%$ in all three directions (Figure 2). Thus, $T$ $=0.09 \mathrm{~s}$ and $f=11.14 \mathrm{~Hz}$ were considered as the lowest period and as the highest frequency of interest for the structure, respectively. Though, as the sixth mode mainly presented vertical displacements along the $\mathrm{Z}$ direction, only the first five modes were considered for dynamic benchmarking between the traditional estimators and the proposed algorithm. Based on the highest period of interest estimated from the eigenvalue analysis, a time step of 0.01 seconds was considered enough to ensure a good resolution for the subsequent THNL analyses. As expected, due to the symmetry of the FE model, the first two vibration modes of the tower are very close-spaced, reading frequency values of $1.79 \mathrm{~Hz}$ and $1.82 \mathrm{~Hz}$, respectively. Both modes present essentially translation components, the first one along the $\mathrm{x}$ direction and the second one along the y direction, and features more than $60 \%$ of effective mass participation each. Thus, they were chosen as representative modes for conducting a point-wise assessment of the 
frequency temporal evolution through time-dependent functions computed via the proposed algorithm, using as input data the numerical accelerations of the nodes featuring the highest displacements for the modes of interest.

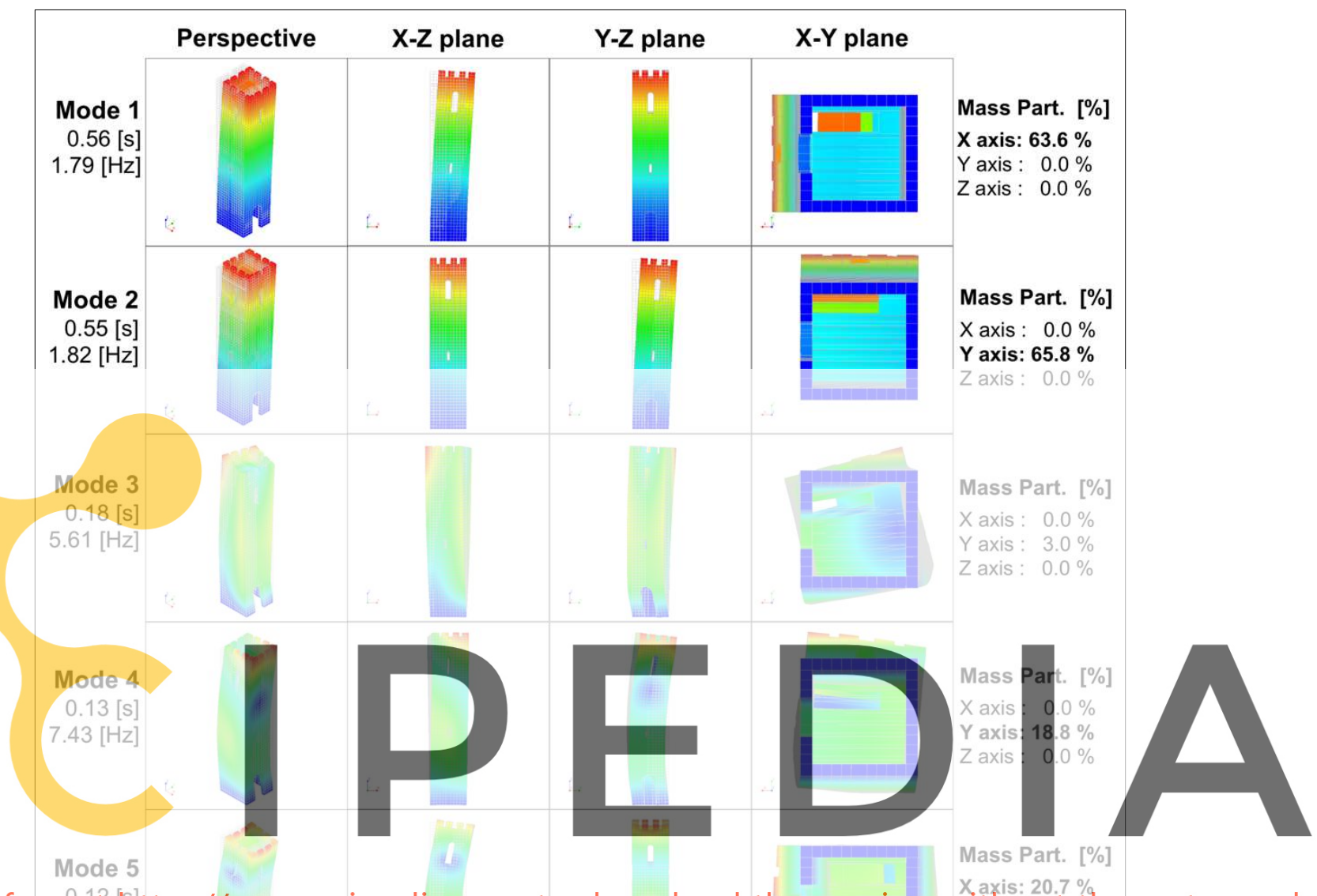

Register for freed.

X.axis: $20.7 \%$

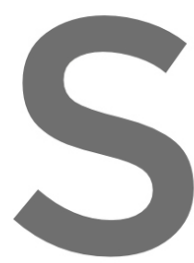

$\mathrm{z}$ axis $0.0 \%$

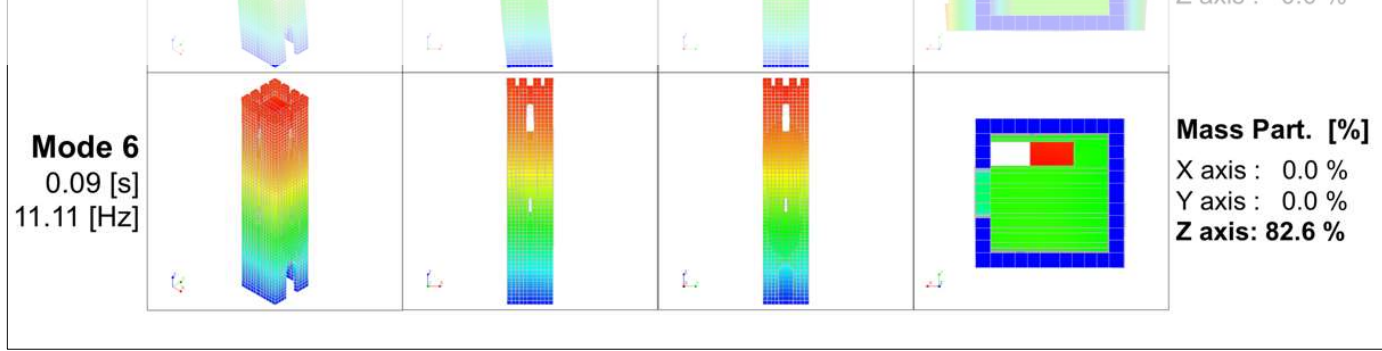

Figure 2: Vibration modes of the tower estimated through the eigenvalue analysis.

\subsection{Time-history analysis with simulated ambient excitations}

Six output-only identification methods available in the commercial software ARTeMIS $^{\circledR}$ were selected as traditional estimators for the feature extraction process, three based on the FDD algorithm and three on the SSI algorithm. In parallel, EMILIA algorithm was run as a Matlab ${ }^{\circledR}$ application. Seventy-one modes with a modal frequency value below the Nyquist frequency 
were found through the FE eigenvalue analysis, thus a six-level MODWPT decomposition was selected for applying the EMILIA algorithm in order to obtain 64 sub-sequences. The results obtained from the different estimators are summarized in Table 2 in terms of modal frequencies of the first ten vibration modes of the tower. By comparing these results against those computed through the eigenvalue analysis, it is observed that SSI-UPC, SSI-UPCX and EMILIA algorithms provide the best results. In particular, EMILIA was capable of identifying most of the searched modes with high accuracy, especially as far as the higher modes are concerned, which results to be a clear advantage for structural health assessment considering that damage is a localized phenomenon that mainly affects high-frequency modes. It is also important to highlight that the frequency values extracted from the EMILIA algorithm are just a statistical approximation, since the main characteristic of the algorithm lies in its capability of computing the dynamic parameters as time-dependent functions.

Table 2: Results for the modal frequency of the first ten modes computed by all estimators

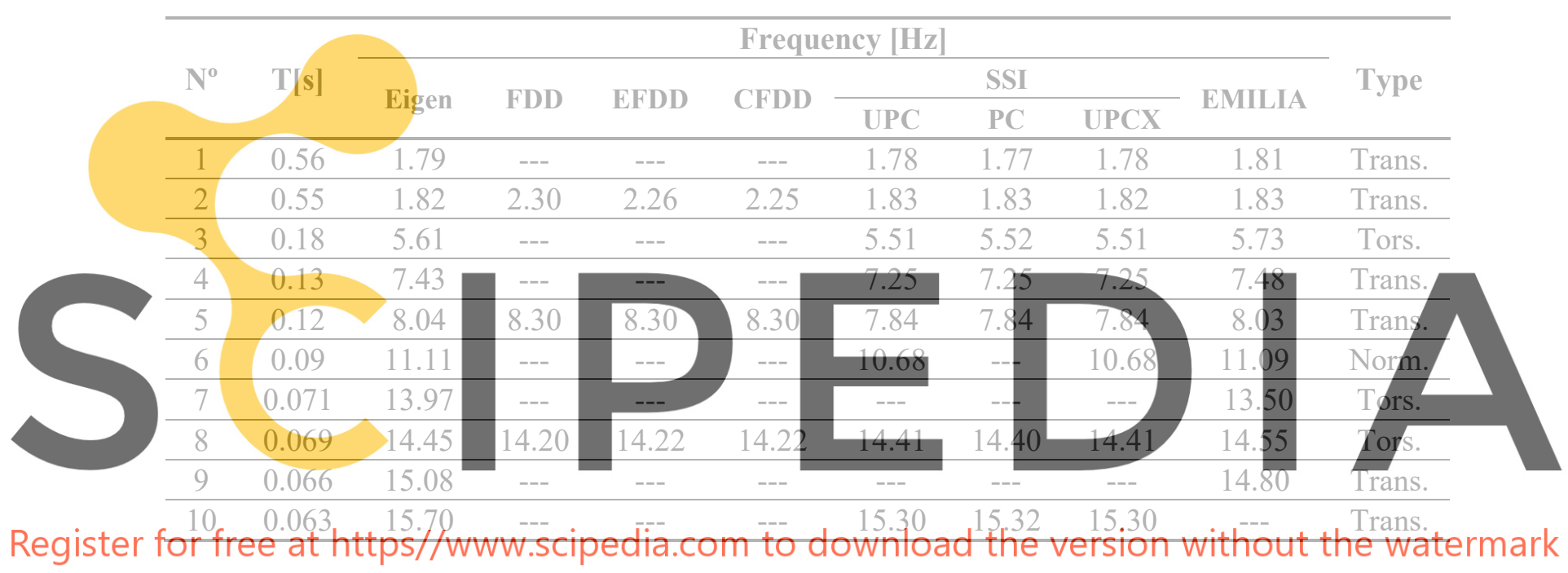

\subsection{Time-history analysis with simulated ambient and reall earthquake excitations}

(left) shows the Hilbert spectra of the first and second modes of the masonry tower plotted as time-dependent functions, allowing to track the evolution of the instantaneous frequency through a 21 minutes THNL analysis, comprising ten minutes of initial ambient excitations randomly applied to different points of the tower, one minute of earthquake excitation applied to the base of the model, and finally ten minutes of additional ambient excitations. Since the analysed data presents non-linearities and high transients, a Daubechies 2 wavelet was selected for conducting the MODWPT decomposition in other to properly identify the abrupt changes in the simulated accelerations. The spectrum corresponding to the first mode (, top-left) clearly shows how the content of the time-dependent frequency function suddenly drops down after 600 seconds (blue continuous line) with the beginning of the earthquake excitation. Further analysis, according to the slope of the first-order polynomial data fits (white-striped lines), also showed a subtle drop down of the global frequency, allowing to infer the occurrence of structural damage in the tower. 

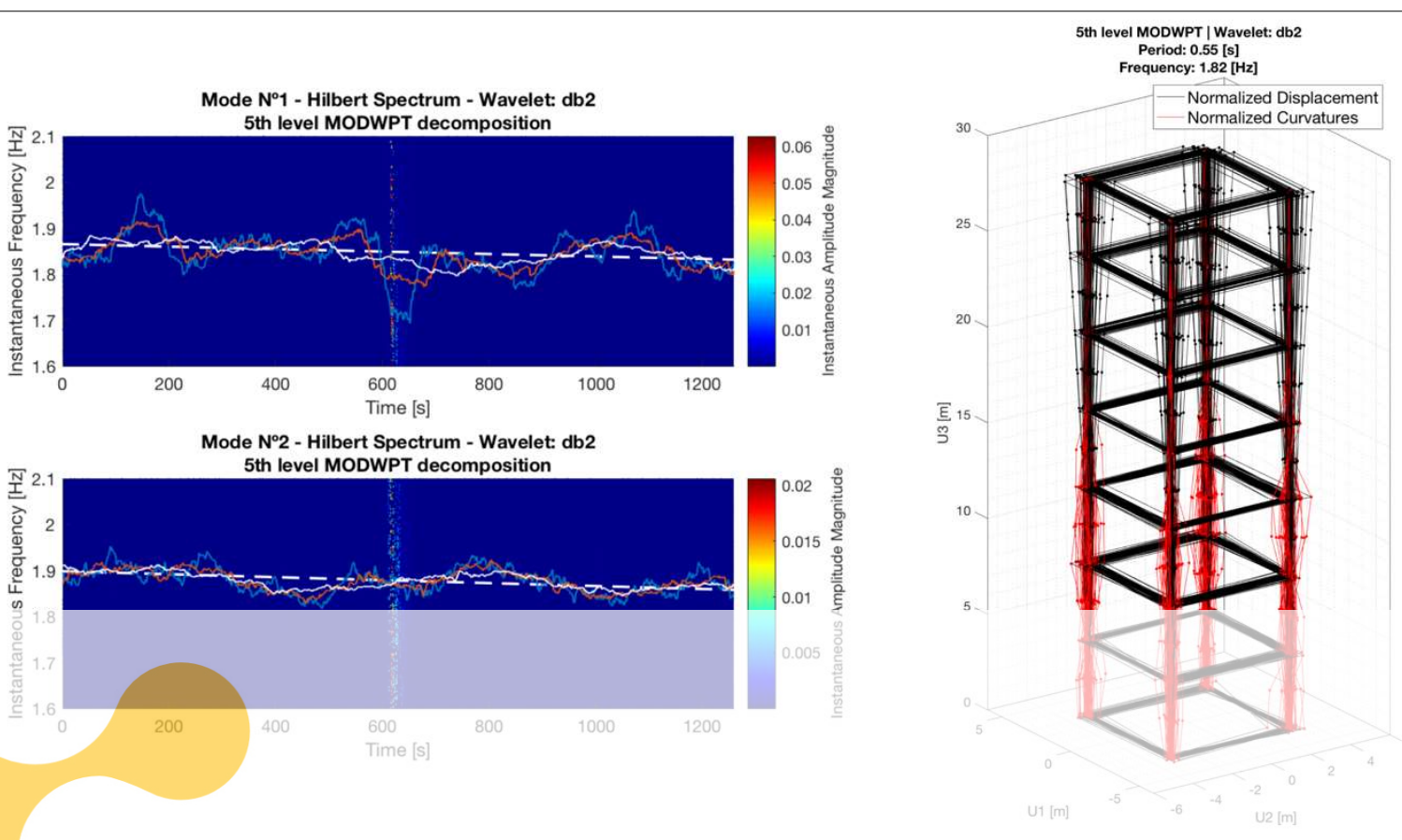

Figure 3: Hilbert spectrum of first (top-left) and second (bottom-left) modes from the idealized masonry tower excited with the 1989 "Loma Prieta" earthquake (all values normalized). White-striped lines show a first-order polynomial fit, continuous blue, red, and white lines show 60s, 120 s and the right, a 3D overlapped plot of several mode shapes computed w
A three-dimensional overlapped plot of several mode shapes

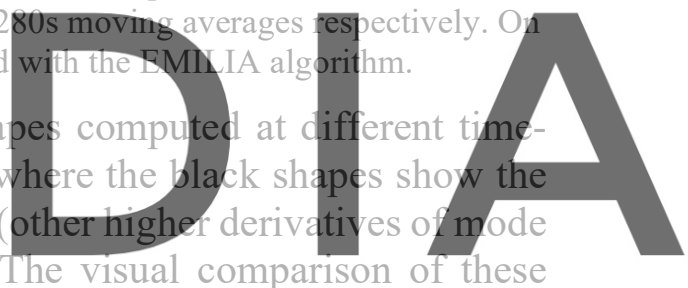

shapes can also be assessed as time-dependant functions). The visual comparison of these

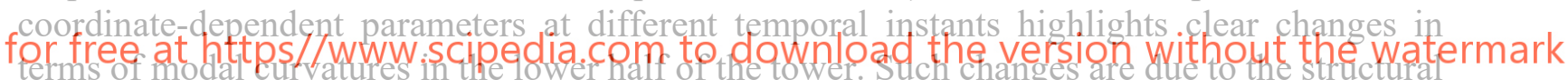
damage induced in the tower by the earthquake and provide indications not only about its approximate location of the damage, but also about the time of occurrence of the damage.

\section{CONCLUSIONS}

The results obtained from the current research allow to draw the following conclusions:

- Wavelet data analysis and Hilbert TFA analysis are rock-solid tools for assessing, decomposing and processing non-linear structural data;

- Wavelet discrete decompositions can appropriately separate MIMO vibration measurements into time-dependent orthogonal functions;

- $\quad$ Hilbert transform instantaneous frequency data can be used to assess temporal changes on structural modal frequencies;

- $\quad$ Displacement mode shape time-dependent functions, and their higher derivatives, are useful tools for the assessment of the structural modal response evolution for health monitoring and damage identification purposes. 


\section{Acknowledgements.}

This work was funded by the National Agency for Research and Development (ANID), through the programme "Doctorado en el Extranjero Becas Chile, Convocatoria 2020" [PhD abroad grants programme, Becas Chile, call 2020], Folio - 72210443 [Sheet number - 72210443]. This work was partially funded by FEDER funds through the Competitiveness Factors Operational Programme (COMPETE) and by national funds through the Foundation for Science and Technology (FCT) within the scope of project POCI-01-0145-FEDER-007633.

\section{REFERENCES}

[1] D. V. Oliveira, L. F. F. Ramos, P. B. B. Lourenço, and J. Roque, "Structural Monitoring of the Monastery of Jerónimos," in 250th Anniversary of the 1755 Lisbon Earthquake International Conference, 2005.

[2] M. J. Morais et al., "A proactive approach to the conservation of historic and cultural Heritage: The HeritageCare methodology," in IABSE Symposium, 2019, pp. 64-71.

[3] M. G. Masciotta, M. J. Morais, L. F. Ramos, D. V. Oliveira, L. J. Sánchez-Aparicio, and D. González-Aguilera, "A Digital-based Integrated Methodology for the Preventive Conservation of Cultural Heritage: The Experience of HeritageCare Project," Int. J. Archit. Herit., 2019.

[4] R. Brincker, "Some Elements of Operational Modal Analysis," Shock Vib., vol. 2014, p. $11,2014$.

[5] N. M. M. Maia, "Extraction of Valid Modal Properties from Measured Data in Structural Vibrations," University of London, 1988.

[6] C. Rainieri and G. Fabbrocino, Operational Modal Analysis of Civil Engineering Structures: An Introduction and Guide for Applications. 2014.

[7] R. Pintelon, P. Guillaume, and J. Schoukens, "Uncertainty calculation in (operational) modal analysis," Mech. Syst. Signal Process., vol. 21, no. 6, pp. 2359-2373, 2007.

[8] X. X. Bao, C. L. Li, and C. B. Xiong, "Noise elimination algorithm for modal analysis," Appl. Phys. Lett., vol. 107, no. 4, Jul. 2015.

[9] S. K. Au, J. M. W. Brownjohn, and J. E. Mottershead, "Quantifying and managing uncertainty in operational modal analysis," Mech. Syst. Signal Process., vol. 102, pp. 139-157, 2018.

[10] R. Brincker, L. Zhang, and P. Andersen, "Modal identification of output-only systems using frequency domain decomposition.," Smart Mater. Struct., vol. 10, no. 3, pp. 441$445,2001$.

[11] R. Brincker, C. E. . Ventura, and P. Andersen, "Damping Estimation by Frequency Domain Decomposition," in Proceedings of IMAC XIX, 2001, pp. 698-703.

[12] J. Rodrigues, R. Brincker, and P. Andersen, "Improvement of frequency domain outputonly modal identification from the application of the random decrement technique," in Proceedings of IMAC XXII, 2004, pp. 92-100.

[13] R. Brincker and P. Andersen, "Understanding Stochastic Subspace Identification," in Proceedings of IMAC XXIV, 2006, pp. 461-466.

[14] P. Van Overschee and B. De Moor, Subspace Identification For Linear Systems. Kluwer 
Academic Publishers, 1996.

[15] B. Peeters and G. De Roeck, "Reference-based stochastic subspace identification for output-only modal analysis," Mech. Syst. Signal Process., vol. 13, no. 6, pp. 855-878, 1999.

[16] F. Ubertini, C. Gentile, and A. L. Materazzi, "Automated modal identification in operational conditions and its application to bridges," Eng. Struct., vol. 46, pp. 264-278, 2013.

[17] M. G. Masciotta, "Damage Identification of Structures based on Spectral Output Signals," University of Minho, 2015.

[18] M. G. Masciotta, L. F. Ramos, P. B. Lourenço, and M. Vasta, "Spectral algorithm for non-destructive damage localisation: Application to an ancient masonry arch model," Mech. Syst. Signal Process., vol. 84, pp. 286-307, 2017.

[19] M. G. Masciotta, L. F. Ramos, P. B. Lourenço, M. Vasta, and G. De Roeck, “A spectrumdriven damage identification technique: Application and validation through the numerical simulation of the Z24 Bridge," Mech. Syst. Signal Process., vol. 70-71, pp. 578-600, 2016.

[20] I. Daubechies, Ten Lectures of Wavelets. Springer-Verlag, 1992.

[21] Y. Wang, Z. He, and Y. Zi, "A demodulation method based on improved local mean decomposition and its application in rub-impact fault diagnosis," Meas. Sci. Technol., vol. 20, no. 2, p. 025704 , Feb. 2009.

[22] I. Provaznik and J. Kozumplik, "Informatics Wavelet transform in electrocardiographydata," Int. J. Med. Inform., vol. 45, no. 1-2, pp. 111-128, 1997.

[23] A. Grossmann and J. Morlet, "Decomposition of hardy functions into square integrable Wavelets of constant shape.," SIAM J. Math. Anal., vol. 15, no. 4, pp. 723-736, 1984.

[24] B. Adhikari et al., "Application of wavelet for seismic wave analysis in Kathmandu Valley after the 2015 Gorkha earthquake, Nepal," Geoenvironmental Disasters, vol. 7, no. $1,2020$.

[25] H. Nyquist, "Certain Topics in Telegraph Transmission Theory," Trans. Am. Inst. Electr. Eng., vol. 47, no. 2, pp. 617-644, 1928.

[26] C. E. Shannon, "Communication in the Presence of Noise," Proc. IRE, vol. 37, no. 1, pp. $10-21,1949$.

[27] A. T. Walden and A. Contreras Cristan, "The phase-corrected undecimated discrete wavelet packet transform and its application to interpreting the timing of events," Proc. R. Soc. London A Math. Phys. Eng. Sci., vol. 454, no. 1976, pp. 2243-2266, 1998.

[28] M. Feldman, Hilbert Transform Applications in Mechanical Vibration, First Edit. Chichester: John Wiley \& Sons, 2011.

[29] A. W. Bowman and A. Azzalini, Applied Smoothing Techniques for Data Analysis: The Kernel Approach with S-PLUS Illustrations, vol. 18. UK, 1997.

[30] E. Ramírez, P. B. Lourenço, and M. D'Amato, "Seismic Assessment of the Matera Cathedral," in 11th International Conference on Structural Analysis of Historical Constructions (SAHC), 2019, vol. 18, pp. 1346-1354. 